\title{
LabScrum: A Case Study For Agility in Academic Research Labs
}

\author{
Lisa May, PhD and Tamara Runyon, CST
}

\section{ABSTRACT}

There are many challenges in the work of academic research labs, such as a lack of established process for planning, competing commitments requiring frequent task switching, and long delays in decisions. Silos of information create opacity of knowledge, and the individual nature of much of the work can create isolation that is demotivating.

We were curious to see if an Agile-based project management approach could provide value in the face of these challenges. The Scrum framework seemed like a good place to start - lightweight, yet with more frequent and shorter feedback loops than before. As an experiment, we implemented Scrum with one lab in the Center for Translational Neuroscience at the University of Oregon. We continue to adapt and evolve as the implementation spreads to other labs and we work with an ever growing number of scientists.

The adaption from Scrum to LabScrum took a great deal of consideration and experimentation given significant differences in goals, constraints, and environment between industry and academia. We named our adaptation LabScrum to reflect the customization for the academic research lab context.

Using LabScrum, lab personnel are seeing increased productivity and increased visibility of short, medium, and long term planning and goals. Personnel are also benefiting from improved graduate student training, increased information sharing/collaboration, and improved social support and positive lab culture.

\section{INTRODUCTION}

LabScrum is the evolution of Scrum used in an academic research setting at The Center for Translational Neuroscience, University of Oregon. The purpose for implementing what has become LabScrum was to increase scientific output as measured by published papers, to share knowledge, and create visibility for faculty members and graduate students (trainees) conducting research. It is interesting to note that as practices evolved, different labs have adopted different patterns for implementing Scrum.

LabScrum has spread rapidly, being adopted in an ever growing number of research labs at the University of Oregon. LabScrum is currently successfully being used in The Center for Translational Neuroscience labs, as well as Biology, Psychology, and Human Physiology research labs. LabScrum evolved from experiments we ran on the application of Scrum in research environments, adapting our approach from a 
more formal Scrum implementation to something that stays true to the values and principles in the Agile manifesto, is based on the Scrum framework, and fits the needs of academic research labs.

There are several traditional issues that occur in research environments which we targeted to resolve with the use of LabScrum, including:

- difficulty in prioritizing competing projects

- lack of systems providing structured planning and vision

- competing commitments and roles (e.g. teaching, research, and clinical work)

- frequent task switching

In addition, dependencies on overburdened faculty mean long delays for decisions, silos of information, and little to no collaboration or knowledge sharing across lab personnel.

The LabScrum adaptation utilizes all the official Scrum events: sprint planning, daily scrum, sprint review, and retrospective. We included the Agile practices of product backlog refinement and release planning as well. These events and practices are implemented in ways that best work for each unique research environment.

The Scrum roles of product owner, scrum master, and development team are adapted, while staying true to the spirit of the purpose of these roles. The artifacts of product backlog, sprint backlog, and product increment also required some adaptation. In particular, ideas around what constitutes a product increment have evolved. In addition, defining metrics that provide value in this context is a continuing work in progress.

\section{Context of ACADEmic Research EnVIRONMENT}

To understand our journey to LabScrum, it helps to understand the context of the research environment. Academic scientific research has two key missions. First, the production of knowledge. In this perspective, manuscripts published in peer-reviewed journals are the main product. Second, to provide training. In this perspective, scientists are the main product. From either of these perspectives, our work exists without the time, scope, or budget constraints that drive projects in a traditional business environment. This lack of constraints brings unique issues that most organizations do not need to solve.

Agile systems in the academic environment have qualitatively different controls than those in the software industry where Scrum originated. Typically, there is no profit motive, and often very little reporting or budget oversight. For example, there is seldom any financial auditing, and no oversight of deliverables or measuring of productivity. Success is measured in terms of papers published and grants awarded, both of which take many years to complete.

\section{Non-Existent Project Management Practices}

A key difference between the traditional business environment and the research environment which factors into our use of LabScrum is the absence of a tradition or culture of project management in academic scientific research. Due to this absence of focus on best practices and standardized process, each lab must "reinvent the wheel", leaving most labs' default process to be inefficient and ineffective. 
Often, this process consists of weekly one-on-one meetings, typically an hour in length, between the faculty member and trainee ${ }^{[1]}$, focused on individual work-in-progress. In addition, there may be a weekly lab meeting with all lab personnel to provide feedback on more finished work (e.g. a conference poster, grant application, manuscript, or conference talk). By default, the faculty member is often the only one who possesses the big picture view of the lab's work; this means they accidentally own the responsibility of repeating information in individual meetings, while all others remain dependent on the faculty member to identify and communicate areas of potential collaboration or knowledge sharing.

\section{Opacity and Few Feedback Loops}

Typically, a trainee's main venue for learning is a one-on-one relationship with their faculty mentor. In this structure, it is difficult to know what others are working on and nearly impossible to identify opportunities for collaboration without the direct involvement of the faculty member. Weekly lab meetings provide limited opportunities for feedback when deliverables are in a near-finished state. In a culture of not working in a shared space (working at home, working in coffee shops, working in offices in separate locations), these meetings are often the only time that lab members see one another face-to-face. During this meeting, trainees receive feedback from their mentor and then go back to struggling in isolation.

\section{INEFFICIENCIES, DeLAYS, AND DEPENDENCIES}

The default system described above is inefficient, causing delays by creating dependence on the faculty member for feedback. Trainees often experience delays in their work, waiting a week or more to receive feedback from their faculty mentor in one-on-one meetings. As one faculty member stated, "I have ten trainees needing feedback from me. I'm always going to be the bottleneck."

\section{LABSCRUM IN ACTION}

Since there is no global process for project management, each lab using LabScrum has made an independent decision to do so. The interest in LabScrum has spread by word of mouth among trainees, formal discussions with faculty at Center events, and even via pleas for help from Twitter! We emphasize flexibility and experimentation in creating successful LabScrum implementations for individual labs and have found that a lab's transition to Agile is helped by hosting a kick-off event with a needs assessment and brainstorming session. This allows lab personnel to customize their LabScrum implementation and begin the process of self-organization. This process results in diversity in LabScrum implementation, while maintaining consistency in core practices.

\section{AdAPTING the ELEMENTS OF SCRUM}

\section{SCRUM EVENTS}

Sprint Length

Sprint lengths vary between one and two weeks depending on the context. Two-week sprints tend to work well in our research environment. One week is generally too short a time period for making meaningful 
progress on research while juggling other commitments such as teaching or taking classes; however, one week sprints can be helpful when an individual is engaged in focused work to meet an impending deadline such as a dissertation or grant submission. Occasionally, an individual working in a lab that uses two-week sprints has expressed a preference for one-week sprints. In these cases, we've been able to meet this need by having the individual "split their sprint" and identify sprint goals for week one and week two seperately. Overall, sprints are generally two weeks in length, and are kept consistent within a particular lab.

\section{Sprint Planning}

Sprint goals are identified and communicated during sprint planning, although there is diversity across labs in how this is executed. Sprint goals are typically organized by individual, since much of the work is independent, and the outcome of sprint planning is usually a series of sprint goals written next to each team member's name on a whiteboard. For example, someone might list completion of a manuscript draft or analysis as a sprint goal, and it is common for individuals to have three or four sprint goals for a twoweek sprint. Sprint plans are often created independently prior to the sprint planning meeting but often adapted based on group discussion of strategy and logistics. This valuable feedback increases success in meeting sprint goals. Most labs spend thirty to sixty minutes per sprint on planning.

\section{The Daily Scrum}

Most lab members juggle time commitments from other roles in addition to their research work. This challenges the feasibility of true daily scrums. Instead, these scrums are more like "scrum of scrums" and tend to work better when held two or three times a week. The fifteen minute timebox and standup method are utilized, but we've found that discussion on progress toward sprint goals is more effective than the traditional "What did you do yesterday? What will you do today? Are there any impediments in your way?" approach since the answers to "What did you do yesterday?" may be work that is not relevant to research like "grading exams". Discussion of progress toward sprint goals is more relevant to the work and is a key method of sharing knowledge across lab personnel. Many labs post sprint goals on a white board in a shared lab space and refer to this board during the daily scrum. Having sprint goals visible and referring to them in the daily scrum encourages focus on priorities that can otherwise get lost in the midst of many competing time commitments.

\section{Sprint Review}

A day or two before the end of the sprint, lab members identify product that could benefit from review, and an agenda is created. Not every sprint goal creates work appropriate for review, so a lab might review and give feedback on one to three items during a ninety minute meeting. This is a critical venue for getting feedback on work in progress and often results in lively, interested discussion that can stretch to fill up a great deal of time if a timebox is not enforced.

\section{Sprint Retrospective}

Constraints on schedule and roles can make sprint retrospectives challenging to arrange, but this meeting has value on multiple levels. In our observations, the sprint retrospective: 
- Helps labs identify improvements to their shared process. For example, in one retrospective, a lab realized that impromptu meetings in the shared lab space were causing distractions and decided to repurpose storage space for impromptu meetings.

- Helps lab personnel identify improvements to their own independent processes. Here, a lab member realized they needed to be more assertive with a collaborator at another University.

- Helps lab personnel provide each other social support which is critical for surviving the pressures of academia. For example, an exhausted new parent received encouragement, validation, and offerings of babysitting.

\section{SCRUM ROLES}

Roles in academic research do not directly parallel scrum master, product owner, or development team members. We have loosely identified the Scrum roles to lab roles paradigm. Each of these is subject to adaptation and change based on changing context from lab to lab.

\section{Product Owner Role}

The faculty member acts as a chief product owner on large lab-wide projects, while trainees act as product owners on their own individual research projects. The faculty member serves as a chief product owner for these individual projects, setting direction, with budget authority and advisory capacity, but not final say in design decisions.

\section{Development Team Role}

While a trainee may be product owner for major portions of a project, responsible for idea generation, design, and planning, they may also act as development team member for execution of the work. Many trainees utilize the efforts of undergraduate and other volunteers to help collect data and then handle the analysis and writing portions independently.

\section{Scrum Master Role}

We see more variation and adaptation with the scrum master role. One lab has a scrum master who splits time about 50/50 with other work, but most labs do not. In other labs, the faculty member takes on scrum master duties, or lab members self-organize to fill this need. For example, one lab has a sheet posted where lab members can sign up to facilitate the sprint retrospective.

\section{AGILE PRACTICES}

\section{Product Backlog Creation and Refinement / Roadmapping and Release Planning}

Backlog creation, refinement, and release planning are organized either around a project or around an individual. These happen on an as-needed basis to facilitate long term strategizing regarding when and what work to prioritize. For example, a student's three-year plan for her dissertation work helped her identify that a faculty member who was providing important statistical training was going to be gone for sabbatical the following year. This knowledge allowed the student to strategize a means of obtaining the necessary training before the faculty member left, thus avoiding a six-month delay to her work. 
We've defined different levels of planning according to the planning horizons. Planning for more than a year in the future roughly equates with high level product roadmap planning, planning within an academic year but further out than two weeks equates with release planning, and planning within two weeks correlates with sprint planning.

\section{Career/Training Plans}

Career/training plans are an element of LabScrum not found in traditional Scrum. These plans are adjacent and related to the product-focused plans discussed above. These plans encourage lab personnel to consider their research work from the perspective of scientific training, not just scientific output. Longterm training goals are articulated and connected to concrete actions that can be part of a sprint. In many labs, faculty and individual trainees meet quarterly to discuss and update training plans.

\section{BENEFITS}

The response to LabScrum has been overwhelmingly positive. Faculty report dramatic time savings, as evidenced by these statements:

- "Turns out there was a project meeting every week I didn't need!"

- "I turn work around in two or three days instead of in two weeks."

- "There's ten more hours in my week I have free to work that I was spending in trainee meetings."

An environment of group learning, shared problem solving, and social support combat isolation and ignorance. LabScrum labs have been described as "ridiculously happy", and trainees report that rapid feedback increases productivity. Trainees get feedback in daily scrums two or three times per week instead of just once weekly in one-on-one meetings, and get formal feedback from faculty (e.g. manuscript draft edits) more quickly due to open time not spent in one-on-one meetings. Long term release plans and training plans allow individuals to identify time-oriented goals, and manuscripts spend less time stuck in unending revisions due to ability to communicate time goals and prioritize progress.

LabScrum fosters better work habits, helps separate planning from execution, and increases the use of effective strategies for planning. Improved and shared documentation increases institutional knowledge and reduces rework. A structure for making competing time commitments visible helps prioritize work. Increased visibility of work-in-progress increases productivity not only through problem solving, but also through shared knowledge. For example, labmates have shared new software discoveries as evidenced by this overheard statement, "There's an (statistical programming) R package for that! You don't have to do it by hand!"

A focus on colocation has made huge impacts on the efficiency and effectiveness of work. A shift from a culture of individual work toward a culture of work in a shared space has been a major benefit from adopting the Scrum framework. This shift has required the creation of appealing work environments, with labs purchasing comfortable furniture and making snacks available. One faculty member shifted entirely to working in the shared lab space with the trainees, which has yielded several positive results. For instance, increased communication - instead of having to compose an email and wait for days to get an answer, one can quickly check in verbally, and/or schedule a time for a more lengthy discussion. In addition, the value of overhearing conversations in this shared work space is not to be discounted. 
Overhearing creates shared knowledge of the lab's work and surfaces colleagues' novel ideas, potential collaborations, and social support.

\section{Challenges AND INSIGHTS}

One enlightening moment in our experiments occurred when we realized that the Scrum events more closely mapped to a "scrum of scrums" across different projects rather than a traditional single scrum team with planning, review, and retrospectives within a single project. Each trainee led their own "projects" and had independent goals. Once this realization occurred, we were able to better adapt the events and roles to reality.

Another major "Ah-ha" moment was introducing release planning and making those plans visible. Long term plans in particular have been a benefit of LabScrum. In some cases, just creating long term plans is highly innovative. In other cases, increasing the visibility of plans has allowed people to seek strategic input from stakeholders, thereby increasing their ability to inspect and adapt. A key realization was the importance of the development of detailed backlogs before the creation of release plans. This shift increased the utility of the planning process by identifying gaps in knowledge and potential barriers early to avoid delays.

One interesting development surfaces when considering metrics. Specifically, considering when metrics create useful knowledge versus when metrics increase reporting load unnecessarily. Since research scientists operate almost exclusively as independent decision agents, often in the context of informal collaborations, metrics for reporting to oversight are not necessary. The metrics of success that are valued are papers published (particularly the quality of the journal and how often other scientists cite the paper) and funding (in the form of federal research grants) awarded.

With the lack of deadlines in most research environments, there is no strong incentive to predict when work will be complete, diminishing the usefulness of traditional Scrum metrics such as velocity. Another reason we've found the metric of velocity and/or capacity to lack utility is the ever changing landscape of external commitments. Instead of being dedicated to a specific team or even project as traditional scrum team members would be, researchers are always juggling time commitments from multiple roles outside of research. The ebb and flow of those commitments makes calculation of capacity inconsequential.

Interestingly enough, however, calculating story points does still have value, but we see the value largely in the self-reflection that is stimulated by the act of assigning points. T-shirt sizing product backlog items are useful for individual strategy and decision making but not for oversight or forecasting future delivery.

\section{ONGOING ADAPTATION}

LabScrum continues to adapt and change to meet the evolving needs of lab personnel. Areas of ongoing interest include developing best practices around managing roles, the potential utility of user stories, productive discussion of impediments, and defining useful metrics. We are working to understand how the funding structure could support dedicated project management staff, or how to work within the funding structure to create LabScrum roles beyond the ad hoc manner in which they occur now. 
To date, user stories have not been adopted in LabScrum; instead, work is currently framed as tasks in the product backlog. User perspectives such as research participants and journal editors/reviewers exist, but researchers report that the time commitment to create user stories for individual work is overly burdensome as it provides little if any added value.

Lastly, there is ongoing discussion among labs about how to structure productive conversation around impediments. In this culture of individualized work and juggling many commitments, common impediments such as task switching and finding time to complete work are systemic in nature. To address this, we are experimenting with a new approach of reporting on needs that the lab can meet. This is a subtle shift in perspective. For example, someone might focus on days of work lost because of a sick child as an (unresolvable) barrier, or instead report on the need for help reprioritizing work because of the lost days of work.

\section{CONCLUSION}

Over the last two years, as we've learned and adapted our Agile practices, we've discovered deep benefits from working with the LabScrum framework. The most notable benefits include reduced time in meetings, increased knowledge sharing, better problem solving through increased collaboration, and more effective work prioritization. Many of our lab personnel have also reported increased "quality of life" using these practices.

By adopting structured feedback loops, utilizing the events in the Scrum framework, and adapting the Scrum roles, LabScrum brings great benefits without greatly increasing overhead. The faculty member enjoys increased time to mentor students, and the added visibility helps with better support and collaboration for the individual trainees. Participants report that LabScrum supports increased productivity in the lifeblood of academic research labs: writing grants, completing necessary research, and publishing papers.

\section{AbOUt THE AuthORS}

DR. LISA MAY is Assistant Director of Operations for the Center for Translational Neuroscience at the University of Oregon where she developed LabScrum as part of her Agile Coaching. Prior to her current role, her research focused on the brain chemistry by which psychology impacts pain perception. She received her $\mathrm{PhD}$ in social neuroscience from the University of Oregon in 2017. More information about LabScrum can be found at abscrum.org and ctn.uoregon.edu/labscrum.

TAMARA RUNYON is a Scrum trainer and enterprise Agile coach with more than 30 years experience in business management and software development. She is the founder and president of Advanced Project Management, Inc. (APMI), as well as Applied Scrum LLC, an Agile consulting business and division of APMI focused on helping organizations adopt and scale Agile practices.

[1]

Note: there are many different types of trainees, including post-doctoral fellows, undergraduate students, graduate students, and research assistants. Regardless of type, we've used the generic term trainee throughout for simplicity. 